ORIENTAL JOURNAL OF CHEMISTRY

An International Open Access, Peer Reviewed Research Journal

www.orientjchem.org
ISSN: 0970-020 X

CODEN: OJCHEG

2020, Vol. 36, No.(2):

Pg. 262-267

\title{
Olax Imbricata-Derived Compounds with $\alpha$-Glucosidase Activity: In vitro Combined Molecular Docking
}

\section{NGA THI VO', HUONG THI MY NGUYEN², TUYET THI ANH NGUYEN ${ }^{3}$ and HAO MINH HOANG ${ }^{1 *}$}

${ }^{1}$ Faculty of Chemical and Food Technology, Ho Chi Minh City University of Technology and Education, Ho Chi Minh City, Vietnam.

${ }^{2}$ Faculty of Chemistry, University of Science, Vietnam National University-Ho Chi Minh City, Ho Chi Minh City, Vietnam.

${ }^{3}$ Faculty of Chemistry, Ho Chi Minh City University of Education, Ho Chi Minh City, Vietnam.

${ }^{\star}$ Corresponding author E-mail: haohm@hcmute.edu.vn

http://dx.doi.org/10.13005/ojc/360207

(Received: February 18, 2020; Accepted: March 20, 2020)

ABSTRACT

Seven compounds (1-7) from Olax imbricata were evaluated the inhibitory activity against $\alpha$-glucosidase. Compound 7 has exhibited stronger activity $\left(\mathrm{IC}_{50}=34.75 \mu \mathrm{g} / \mathrm{mL}\right)$ than that of acarbose $\left(\mathrm{IC}_{50}=187.50 \mu \mathrm{g} / \mathrm{mL}\right)$, a positive control. The molecular docking results revealed that compound 7 strongly inhibits $\alpha$-glucosidase due to the formation of a stable ligand- $\alpha$-glucosidase complex via hydrogen bonds and hydrophobic interactions. The favorable complex formation of 7 with target enzyme gave the more negative docking score-DS $(-28.52 \mathrm{kcal} / \mathrm{mol})$ than that of acarbose $(-23.01 \mathrm{kcal} / \mathrm{mol})$. A slight inhibitory activity was observed for $4\left(\mathrm{IC}_{50}=217.50 \mu \mathrm{g} / \mathrm{mL}\right.$, DS $=-21.35$ $\mathrm{kcal} / \mathrm{mol}$ ) while compounds 1, 2, 3, 5 and 6 giving $\mathrm{IC}_{50}$ values $>256 \mu \mathrm{g} / \mathrm{mL}$ and less negative binding energies have shown no effects on the $\alpha$-Glucosidase inhibition.

\section{Graphical abstract}

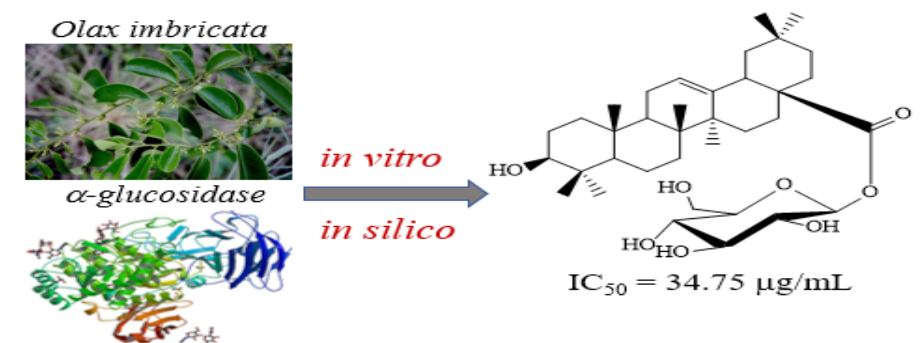

Keywords: Diabetes, Olax imbricata, $\alpha$-Glucosidase, Acarbose, Molecular docking.

This is an Open Access article licensed under a Creative Commons license: Attribution 4.0 International (CC- BY). Published by Oriental Scientific Publishing Company @ 2018 


\section{INTRODUCTION}

Diabetes, characterized by a high blood glucose concentration over time, leads to amputation, blindness and reduced renal function. Diabetes resulting from defects in insulin, a hormone produced from the pancreas, occurs either the pancreas not producing enough insulin (insulindependent diabetes) or the cells not responding to the insulin produced (insulin resistance) ${ }^{1}$. Insulin regulates the metabolism of proteins by promoting the absorption of carbohydrates, especially glucose from the blood into cells. Therefore, the relative insulin insufficiency (high glucose levels) in the blood would damage to the metabolic context of the organism. In order to maintain normal glucose levels in the blood, the inhibition of the glucose production from carbohydrates could be taken into account. $\alpha$-Glucosidase, an enzyme digestion, is responsible for the hydrolysis of carbohydrates in the small intestine. $\alpha$-Glucose released by the activity of this enzyme on 1,4- $\alpha$-glycosidic bonds of carbohydrates, and thus a high glucose level is found in the blood ${ }^{2-4}$. As a result, this enzyme was considered as an effective target for the diabetic therapy. There are few $\alpha$-Glucosidase inhibitors in the clinical practice but they have limited efficacy and are associated with side effects. Thus, new effective and safe drugs such as various bioactive components in plants are further explored for the development of more useful $\alpha$-Glucosidase inhibitors.

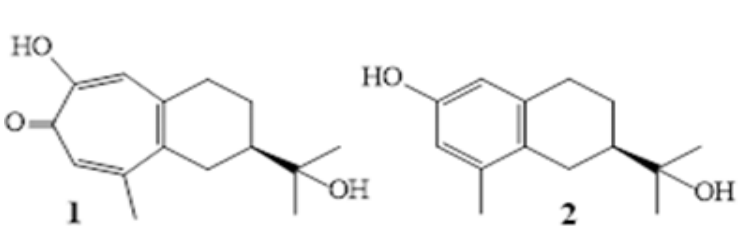<smiles>COc1cc(O)cc(OC)c1OC</smiles>

5<smiles>COc1cc(C(=O)OCC2COC(Oc3c(OC)cc(O)cc3OC)C(O)C(O)C2O)cc(OC)c1O</smiles>

The genus Olax belongs to Olacaceae and consists of a huge number of bioactive metabolites. Natural products isolated from this genus showed various biological functions including anticancer, inflammatory, Alzheimer's diseases ${ }^{5-7}$. To the best of our updates there was a report on the $\alpha$-Glucosidase inhibitory potential of Olax nana. The half maximal inhibitory concentration $\left(\mathrm{IC}_{50}\right)$ values of the $\alpha$-Glucosidase inhibitory assay for the methanolic extract and acarbose used as positive control were $639.89 \mu \mathrm{g} / \mathrm{mL}$ and $61.19 \mu \mathrm{g} / \mathrm{mL}$, respectively ${ }^{8}$. In order to continuously search and optimize structure of compounds showing the anti-diabetic activity from the genus Olax, the In vitro and molecular docking results involving seven compounds isolated from Olax imbricata with $\alpha$-Glucosidase were reported in our present work.

\section{MATERIALS AND METHODOLOGY}

\section{Natural compounds and their chemical structures}

The isolation and structure elucidation of seven compounds (1-7, 1-4 are novel) from Olax imbricata have been reported in details in our previous publications ${ }^{9-12}$. Pure materials were used for In vitro experiments with $\alpha$-Glucosidase while their chemical structures (Fig. 1) selected for molecular docking to analyze the binding interactions with receptor.<smiles>COc1ccc(C)c2c1CCC([C@](C)(O)CO)C2</smiles>

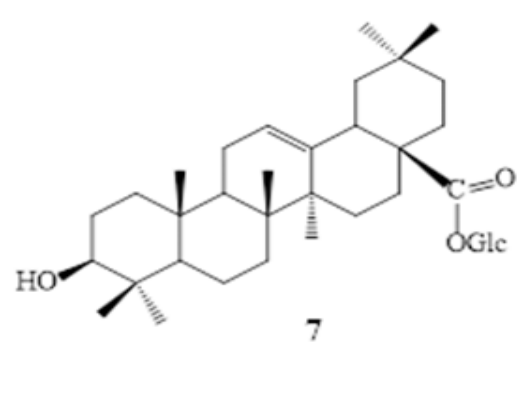

Fig. 1. Chemical structures of seven natural compounds (1-7) extracted from Olax imbricata were subjected to the $\alpha$-Glucosidase inhibitory assay and molecular docking. Glc refers to a glucose unit connected aglycone through an ether linkage 


\section{$\alpha$-Glucosidase inhibition assay}

The $\alpha$-glucosidase inhibitory assay was carried out following the published procedure described by Hakamata with some modifications ${ }^{13}$. Samples were dissolved in dimethylsulfoxide (DMSO, Aldrich 99.9\%) at different concentrations for the $\alpha$-glucosidase inhibitory assay. A total reaction volume of $200 \mu \mathrm{L}$ was added to each well of the 96-well plate. The reaction solution containing $40 \mu \mathrm{L} 0.1 \mathrm{M}$ phosphate buffer solution at $\mathrm{pH} 6.8$, $25 \mu \mathrm{L}$ a-glucosidase 0.2 unit/mL (CAS No 900142-7, from Saccharomyces cerevisiae, Aldrich), $10 \mu \mathrm{L}$ sample solution and $25 \mu \mathrm{L} p$-nitrophenyl $\alpha$-D-glucopyranoside $2.5 \mathrm{mM}$ (Aldrich 99\%) was added to each well and mixed. Subsequently, the mixture was incubated for $30 \mathrm{~min}$ at $37^{\circ} \mathrm{C}$. Finally, the reaction solution was terminated by adding 100 $\mu \mathrm{L}$ of $0.2 \mathrm{M}$ sodium carbonate solution. For the negative control, the sample was replaced with the buffer solution and acarbose was used as positive control. Experiments were performed in triplicate. The absorbance of the sample was measured with a BIOTEK microplate reader at $410 \mathrm{~nm}$. The percent inhibition I (\%) of $\alpha$-Glucosidase inhibitory activity was calculated using the equation (1):

$I(\%)=\frac{A_{(\text {blank })}-A_{(\text {sample })}}{A_{(\text {blank })}} \times 100 \%$

Here, $A_{\text {(blank) }}$ and $A_{\text {(sample) }}$ are the absorbances of the blank and sample, respectively.

The $I_{50}$ (the half maximal inhibitory concentration) was determined by calibration curve equation of the percent inhibition versus sample concentration using Tablecurve software. $I_{50}$ values are the mean values of three experiments.

\section{Preparation of $\alpha$-Glucosidase and ligands for molecular docking}

The crystal structure of $\alpha$-Glucosidase was retrieved from the protein data bank (PDB: $3 \mathrm{~W} 37)^{14}$. The chain and binding sites of target enzyme were prepared by using Molecular Operating Environment 2008.10 (MOE 2008.10) software. In LigX tool, the 3D structure was loaded into MOE, the co-crystallized ligand acarbose with $\alpha$-Glucosidase receptor was selected as a reference ligand. The three-dimensional (3D) protonation was carried out to add protons and partial charges to the system. Sequentially, the receptor atoms were added tethers and performed an energy minimization of the ligand and pocket residues. The unbound water molecules surrounding the active sites were deleted in the next step and finally, the prepared $\alpha$-Glucosidase was saved for molecular docking as * ${ }^{*}$.pdb. Prior to docking, the $3 \mathrm{D}$ structures of seven compounds were drawn in MOE and minimize energy to obtain the conformation in the lowest energy by using SYBYL-X 2.0. The energy minimization procedure was ended when an energy change between two consecutive conformations was $0.0001 \mathrm{~kJ} / \mathrm{mol}$. Each ligand was saved as *.mol2 for further analysis.

Molecular docking was carried out to analyze the binding interactions of ligands and $\alpha$-glucosidase by using LeadIT 2.0.2. In order to dock ligands into the prepared $\alpha$-glucosidase, the binding sites of receptor were initially defined by selecting acarbose as a reference ligand and a binding pocket including amino acids within radius of $6.5 \AA$. The ligands were loaded from the docking library. The top-ranked conformations of each ligand were 10, i.e., the most negative docking score was ranked as 1. The maximum number of solutions per iteration and fragmentation are 1000 and 200, respectively.

\section{RESULTS}

\section{Redocking}

The docking procedure and parameters need to be validated by redocking. The native pose of acarbose, a co-crystallized ligand with $\alpha$-glucosidase, was separated from the receptor and redocked to its binding sites. Fig. 2 gave a superposition between the native binding pose of acarbose from an acarbose- $\alpha$-glucosidase complex crystal structure with the one extracted from the same complex. The root mean square deviation (RMSD) value of redocking was 0.8469 .

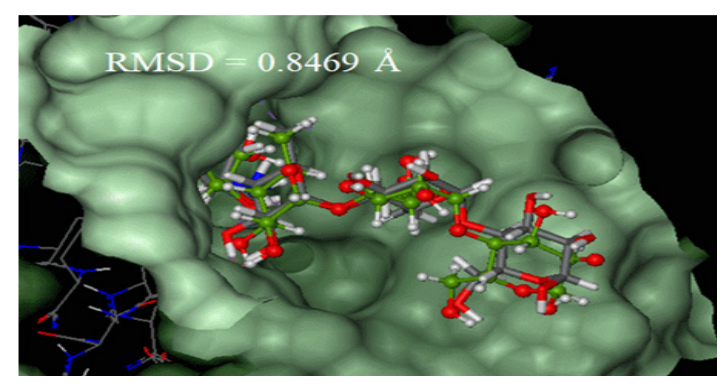

Fig. 2. A superposition of an original acarbose pose (sticks in green, atoms were exhibited in balls: carbon: green, oxygen: red, nitrogen: blue, hydrogen: white) and a co-crystallized acarbose one (sticks in grey) separated from $\alpha$-Glucosidase 
$\alpha$-Glucosidase inhibitory activities and molecular docking

To explore the potential antidiabetic activity of the metabolites purified from Olax imbricata, In vitro screening of $\alpha$-Glucosidase inhibition was performed. Acarbose, an oral antidiabetic drug due to its inhibitory activity against $\alpha$-Glucosidase, was used as positive control. Table 1 gave $\mathrm{IC}_{50}$ values of seven compounds compared to that of acarbose.

To comprehend the observed $\alpha$-Glucosidase inhibitory activities of isolating compounds, molecular docking calculations were performed to explore the binding interactions of the target enzyme and pure ligands. To compare to acarbose, the co-crystallized ligand acarbose with $\alpha$-glucosidase receptor (PDB: 3W37) was selected as template for molecular docking. The number of intermolecular hydrogen bonds, hydrophobic interactions and the free binding energies (docking scores-DS) of stable ligand- $\alpha$-glucosidase complexes were also calculated (Table 1).

Table 1: $I_{50}$ values with their standard deviations and docking scores of seven natural compounds isolated from Olax imbricata and acarbose toward $\alpha$-Glucosidase

\begin{tabular}{ccccc}
\hline Compounds & $\begin{array}{c}\text { Docking score } \\
(\mathrm{kcal} / \mathrm{mol})\end{array}$ & $\begin{array}{c}\text { Number of } \\
\text { hydrogen bonds }\end{array}$ & $\begin{array}{c}\text { Number of hydrophobic } \\
\text { interactions }\end{array}$ & $\mathrm{IC}_{50} \pm \mathrm{SD}(\mu \mathrm{g} / \mathrm{mL})$ \\
\hline 1 & -9.04 & 03 & 04 & $>256$ \\
2 & -10.29 & 02 & 07 & $>256$ \\
3 & -20.11 & 07 & 08 & $>256$ \\
4 & -21.35 & 07 & 08 & $217.50 \pm 13.94$ \\
5 & -12.87 & 07 & 06 & $>256$ \\
6 & -11.78 & 04 & 09 & $>256$ \\
7 & -28.52 & 07 & 06 & $34.75 \pm 0.81$ \\
Acarbose & -23.01 & 13 & 10 & $187.50 \pm 3.39$ \\
\hline
\end{tabular}

To reveal possible intermolecular interactions of ligands and amino acid residues surrounding the active sites, two-dimensional (2D) binding
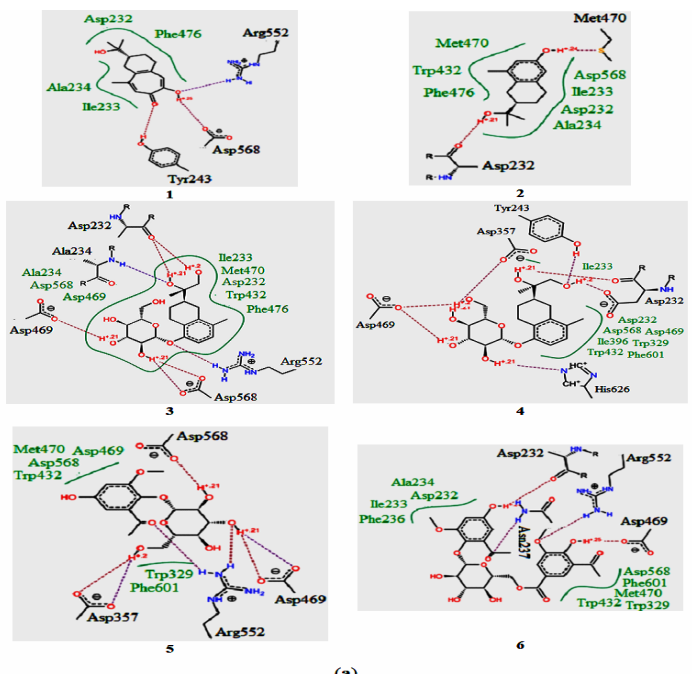

(a) interactions of docking poses of (1-7) and hot spots of $\alpha$-Glucosidase were explored (Fig. 3a-b). The ligands formed complexes with the target enzyme.
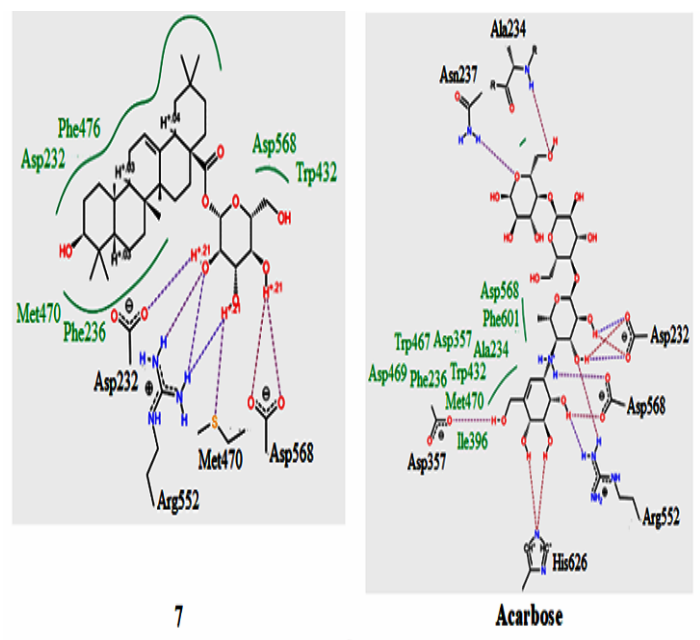

(b)

Fig. 3. Two-dimensional binding interactions of docking poses of (1-7), acarbose and active sites of $\alpha$-Glucosidase. Red dash lines depicted hydrogen bonds while green curves gave hydrophobic interactions between ligands and amino acid residues.

\section{DISCUSSION}

The reasonable potential docking model was confirmed via the root mean square deviation (RMSD) value. The RMSD parameter refers to the deviation between docked pose and the respective 
crystal conformer of the ligand. The RMSD value less than $2 \AA$ suggests that the ligand-protein docking protocol is satisfactory for pose predictions ${ }^{15-17}$. The exhibited RMSD value of $0.8469 \AA$ confirmed that the docking program is applicable to find the optimal conformation and binding affinity of the inhibitors within the $\alpha$-Glucosidase binding pocket.

The rank order of potential activities according to the $\mathrm{IC}_{50}$ values, including acarbose is 1 , 2, 3, 5, $6<4<$ acarbose $<7$. Compound 7 exhibited a much lower $I_{50}$ value $(34.75 \mu \mathrm{g} / \mathrm{mL})$ than that of acarbose $(187.50 \mu \mathrm{g} / \mathrm{mL})$. The $I_{50}$ values of 1,2 , $3,5,6$ are more than $256 \mu \mathrm{g} / \mathrm{mL}$. These structures displayed no effects on the inhibitory activities while compound $4(217.50 \mu \mathrm{g} / \mathrm{mL})$ exhibited a decent activity against $\alpha$-Glucosidase when compared to acarbose.

The inhibition activity basically correlates with the number of interactions which numerically described by docking scores. The docking score calculation (free binding energy) was based on non-covalent intermolecular interactions between ligand-protein such as hydrogen bonds and hydrophobic contacts ${ }^{18}$. A much negative docking score indicates a stable ligand-protein complex and thus, a likely binding interaction. The free binding energies of forming complexes were negative and less than $-9.04 \mathrm{kcal} / \mathrm{mol}$. The results of Table 1 and Fig. 3 can be assumed that the lowest $I C_{50}$ $(34.75 \mu \mathrm{g} / \mathrm{mL})$ value, i.e., the most inhibitory activity of 7 was mainly attributable to the formation of a stable ligand-enzyme complex. This led to be the most negative binding energy $(-28.52 \mathrm{kcal} / \mathrm{mol})$ of 7 compared to the energies of the docked natural compounds. Interestingly, the inhibitory activity of 7 is much higher than that of the reference. The number of established interactions can be relevant in this case. The residues of Asp-232, Arg-552, Asp-568 are located in the active site to form hydrogen bonds with 7. The sulfur atom of Met-470 formed hydrogen bond with hydroxy group of glucose moiety. These residues were found to form hydrogen bonds with acarbose with the exception of Met-47019, 20. In addition, the aglycone containing ring structure is enclosed by hydrophobic contacts with Phe-236, Met-470, Asp-232 and Phe-476. These interactions enhance the stability of a ligand-enzyme complex and thus, reduce free binding energy. Theoretically, molecular docking predicts the preferred orientation of one molecule within active site of target enzyme, i.e., pharmacokinetics of substance has not been taken into account. Here, a synergy of pharmacokinetics and ligand-enzyme complex stability can be responsible for a promising inhibition of 7 toward $\alpha$-Glucosidase.

The binding energies of docked $3(-20.11$ $\mathrm{kcal} / \mathrm{mol})$ and $4(-21.35 \mathrm{kcal} / \mathrm{mol})$ were found to be negative. Compound 3 differs from 4 only at the absolute configuration at $\mathrm{C}-11$. The different $3 \mathrm{D}$ orientations of groups at $\mathrm{C}-11$ in space caused no more binding modes in the active pocket. However, compound 4 at the $\mathrm{IC}_{50}$ value of $217.50 \mu \mathrm{g} / \mathrm{mL}$ exhibited a better activity against $\alpha$-Glucosidase enzyme while 3 (> $256 \mu \mathrm{g} / \mathrm{mL})$ was inactive. The $\mathrm{IC}_{50}$ values of compounds 1, 2, 5 and 6 (> 256 $\mu \mathrm{g} / \mathrm{mL}$ ) followed a similar trend. They did not exhibit any inhibitory activities against $\alpha$-Glucosidase. The results can be mainly attributable to the unfavorable formation of the ligand- $\alpha$-glucosidase complexes regardless of possible interactions. Therefore, the binding energies of docked structures were found to be less negative $(-9.04-12.87 \mathrm{kcal} / \mathrm{mol})$.

\section{CONCLUSION}

In conclusion, seven compounds isolated from Olax imbricata were subjected to $\alpha$-Glucosidase inhibitory assay and molecular docking. The results of natural compounds were compared with acarbose as the reference drug. Compound 7 exhibited a promising inhibitory activity against $\alpha$-glycosidase with a much lower $\mathrm{IC}_{50}$ value than that of acarbose. Molecular docking was conducted and provided insights of the active sites in which a ligand-enzyme complex can be formed. The simulation results showed that compound 7 containing a glucose moiety connected to terpene skeleton created efficient interactions with amino acid residues surrounding the binding site. The lowest binding energy of this saponin is responsible for the high inhibitory activity. The other compounds 1, 2, 3, 5 and 6, which except for 4 , did not show the $\alpha$-Glycosidase inhibition. They might form unfavorable ligand-enzyme complexes in the active site. The study provided new naturally structure insights for the development of novel $\alpha$-Glycosidase inhibitors, which differ from the commonly used sugar mimetics. 


\section{ACKNOWLEDGEMENT}

Ho Chi Minh City University of Technology and Education is gratefully acknowledged by providing the facilities necessary to complete this project. The authors thank Prof. Phung Phi Kim Nguyen for helpful comments.

\section{Conflicts of interest}

No potential conflict of interest was reported by the authors.

\section{REFERENCES}

1. Sonksen, P.; Sonksen, J. Br. J. Anaesth., 2000 85(1), 69-79.

2. Bruni, C. B.; Sica, V.; Auricchio, F.; Covelli, I. Biochim. Biophys. Acta., 1970, 212(3), 470-477.

3. Van de Laar, F. A. Vasc. Health Risk Manag., 2008, 4(6), 1189-1195.

4. Kim, Y. M.; Jeong, Y. K.;Wang, M. H.; Lee, W.Y.; Rhee, H. I. Nutrition., 2005, 21(6), 756-761.

5. Saliu, J. A.; Olabiyi, A. A. Pharm. Biol., 2018, 55(1), 252-257.

6. Popoola, T. D.; Awodele, O.; Omisanya, A.; Obi, N.; Umezinwa, C.; Fatokun, A. A. J. Ethnopharmacol., 2016, 194, 440-449.

7. Okoye, F. B.; Sawadogo, W. R.; Sendker, J.; Aly, A. H.; Quandt, B.; Wray, V.; Hensel, A.; Esimone, C. O.; Debbab, A.; Diederich, M. J. Ethnopharmacol., 2015, 176, 27-34.

8. Ovais, M.; Ayaz, M.; Khalil, A. T.; Shah, S. A.; Jan, M. S.; Raza, A.; Shahid, M.; Shinwari, Z. K. BMC Complement Altern. Med., 2018, 18(1), 13 pages.

9. Nguyen, T. M. H.; Vo, T. N.; Huynh, T. M. S.; Do, T. M. L.; Aree, T.; Tip-pyang, S.; Phan, D. C. T.; Nguyen, T. T.; Nguyen, K. P. P. Fitoterapia. 2019, 132, 1-6.

10. Huynh, T. M. S.; Nguyen, T. M. H.; Nguyen, K. P. P.; Vo, T. N. Vietnam Journal of Chemistry.,
2018, 56(3E12), 336-342.

11. Huynh, T. M. S.; Vo, T. N.; Nguyen, K. P. P. Vietnam Journal of Chemistry., 2015, 53(6e3), 81-84.

12. Vo, T. N.; Huynh, T. M. S.; Nguyen, T. M. H.; Duong, T. H.; Nguyen, K. P. P. Science \& Technology Development Journal., 2019, 22(3), 324- 334

13. Hakamata, W.; Kurihara, M.; Okuda, H.; Nishio, T.; Oku, T. Curr. Top. Med. Chem., 2009, 9(1), 3-12.

14. Tagami, T.;Yamashita, K.; Okuyama, M.; Mori, H.; Yao, M.; Kimura, A. J. Biol. Chem., 2013, 288(26), 19296-19303.

15. Kitchen, D. B.; Decornez, H.; Furr, J. R.; Bajorath, J. Nat. Rev. Drug Discov., 2004, 3, 935-949.

16. Plewczynski, D.; Ła'zniewski, M.; Augustyniak, R.; Ginalski, K. J. Comput. Chem., 2011, 32, 742-755.

17. Bell, E. W.; Zhang, Y. J. Cheminformatics., 2019, 11(40), 9.

18. Böhm, H. J. J Comput Aid Mol Des., 1998, 12(4), 309-323.

19. Chiba, S. Biosci. Biotechnol. Biochem., 1997, 6(18), 1233-1239.

20. Bischoff, H. Clin Invest Med., 1995, 18(4), 303-311. 